\title{
Cricket in Afghanistan: Perspective of a Surgeon and a Sports Enthusiast
}

\section{Emal Wardak}

The growth of cricket in Afghanistan is a remarkable story. The country has moved from virtually no involvement in the game to a good level of international success in only 10 years.

The Asian Cricket Council (ACC) has described Afghanistan as the rising star of Asian cricket. The International Cricket Council (ICC) has said: Afghanistan can now rightly consider itself as one of the leading Associate nations.

The game, now the fastest growing sport in the country, has captured the hearts and minds of a huge percentage of the population. Interest and participation continue to have a spiraling growth as the National Team adds success to success. These successes have also captured the imaginations of the international cricketing world and the world media. The battle of the young players with little or no support and infrastructure, often against almost insurmountable odds, is a story being told and retold again and again.

The majority of the actors in this fascinating story are young Afghans from

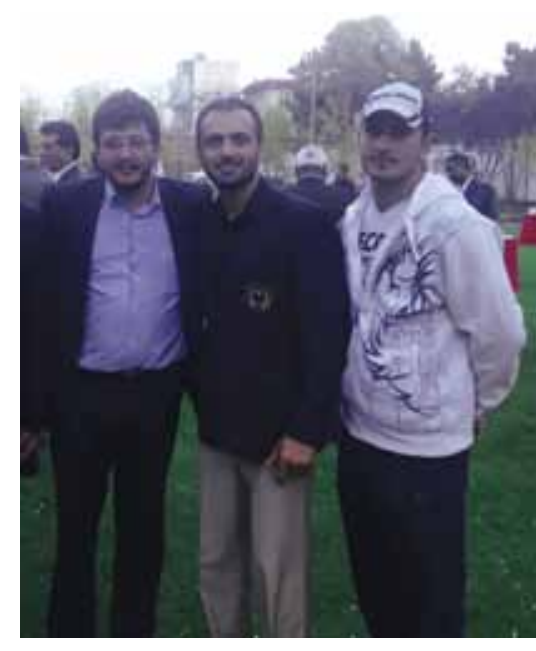
poor families who grew up in refugee camps or small villages. Some had rarely been outside their villages until they began playing cricket.

The one common factor among them all was their passion for cricket and their dream of playing the game at the international level.

Comparing our cricket team with international teams in terms of resources and funds shows that they are among the poorest in the region; despite all the struggles and hurdles they are so passionate that nothing can stop them.

Medical issues and application of scientific training protocols to the game in my country are abysmal. Being a new team, with limited maturity and limited scientific and medical technical backup, many potential problems often beset these cricketers; not having proper training systems many of them get frequently injured, and rehabilitation options are less than optimal. There are no specialized sports clinics, or scientifically applied facilities for the development of bowling actions are rehab techniques. To add to the woes even player insurance is often non-existent, which quite commonly make them suffer or even get disabled because of neglected injuries or unrecognized problems. I myself operated Team Captain Nawrooz Mangal for a traumatic mallet finger (he is a considered world class fast bowler). Luckily he is all right and back to the business of bowling, but this is one example of a player in the capital who presented to a specialist center on his own. However, the scenario in the rest of the country is bleak, and the necessity of a sports medicine clinic, and trained rehab facilities available to all levels of cricketers is being felt by both the players as well as the administrators.

Afghanistan and its cricketers would appreciate any help rendered, as currently most of our players go to India for treatment; this has happened due to the recent improvement in the economy and a better exposure to our team in International competitions, with an emerging groundswell of support. Any equipment, medical literature, access to knowledge updates for trainer, coaches, physios and even doctors would be welcomed. My team and I am willing to act as a conduit for the ingress of knowledge to upgrade cricket in our country from the scientific point of view; the game is played by sportspersons, but improvements are needed in the science behind the game.

Consultant

Department of Orthopedics and Traumatology, Wazir Akbar Khan Government Hospital, Kabul, Afghanistan

Corresponding Author: Emal Wardak, Consultant, Department of Orthopedics and Traumatology, Wazir Akbar Khan Government Hospital, Kabul, Afghanistan, Phone: 93707034241, e-mail: emalpgi@gmail.com 
The current all professional Cricket team does not have access to professionally trained sport medicine doctors and physiotherapists; they do have a team doctor who is MBBS with basic medical skills, but with limited training in sports medicine.

Comparing ourselves with India, Pakistan, and the rest of the world, we are at much lower position to safeguard our players, or to apply scientific methods for their training, individuals like myself, trained abroad, are working on developing some sort of sports medicine clinic, and we are encouraging some training for the staff in our neighboring countries.

And this is the best opportunity for requesting help from those who have already passed our stage of development, to give us guidance and whatever scientific inputs they can. 